\title{
Lusioersily
}

\section{Maternal Gestational Immune Response and Autism Spectrum Disorder Phenotypes at 7 Years of Age in the Seychelles Child Development Study}

Irwin, J. L., Yeates, A. J., Mulhern, M. S., Mc Sorley, E. M., Strain, J. J., Watson, G. E., Grzesik, K., Thurston, S. W., Love, T. M., Smith, T. H., Mruzek, D. W., Shamlaye, C. F., Monthy, C., Myers, G. J., Davidson, P. W., \& van Wijngaarden, E. (2019). Maternal Gestational Immune Response and Autism Spectrum Disorder Phenotypes at 7 Years of Age in the Seychelles Child Development Study. Molecular Neurobiology, 56, 5000-5008. https://doi.org/10.1007/s12035-018-1424-y

Link to publication record in Ulster University Research Portal

Published in:

Molecular Neurobiology

Publication Status:

Published (in print/issue): 01/07/2019

DOI:

10.1007/s12035-018-1424-y

\section{Document Version}

Author Accepted version

\section{General rights}

Copyright for the publications made accessible via Ulster University's Research Portal is retained by the author(s) and / or other copyright owners and it is a condition of accessing these publications that users recognise and abide by the legal requirements associated with these rights.

\section{Take down policy}

The Research Portal is Ulster University's institutional repository that provides access to Ulster's research outputs. Every effort has been made to ensure that content in the Research Portal does not infringe any person's rights, or applicable UK laws. If you discover content in the Research Portal that you believe breaches copyright or violates any law, please contact pure-support@ulster.ac.uk. 
Maternal gestational immune response and autism spectrum disorder phenotypes at 7 years of age in the Seychelles Child Development Study

Jessica L Irwin, Ph.D. ${ }^{a}$, Alison J Yeates, Ph.D. ${ }^{\mathrm{b}}$, Maria S Mulhern, Ph.D. ${ }^{\mathrm{b}}$, Emeir M McSorley, Ph.D. ${ }^{\mathrm{b}}$, JJ Strain Ph.D. ${ }^{b}$, Gene E Watson, D.D.S., Ph.D. ${ }^{a}$, Katherine Grzesik, Ph.D. a, Sally W Thurston, Ph.D. a, Tanzy M Love, Ph.D. ${ }^{a}$, Tristram H Smith, Ph.D. ${ }^{a}$, Daniel W Mruzek, Ph.D. ${ }^{a}$, Conrad F Shamlaye, M.D. ${ }^{c}$, Catriona Monthy, M.A. ${ }^{\text {, }}$ Gary J Myers, M.D. ${ }^{a}$, Philip W Davidson, Ph.D. ${ }^{a}$, Edwin van Wijngaarden, Ph.D. ${ }^{\mathrm{a}}$

${ }^{\text {a }}$ University of Rochester School of Medicine and Dentistry, 601 Elmwood Avenue, Rochester, NY 14642

${ }^{\mathrm{b}}$ Nutrition Innovation Centre for Food and Health (NICHE), Ulster University, Cromore Road, Coleraine, BT52 $1 \mathrm{SA}, \mathrm{Co}$. Londonderry, UK

${ }^{\mathrm{c}}$ Ministry of Health, Box 52, Mahé, Republic of Seychelles

${ }^{\mathrm{d}}$ Ministry of Education \& Human Resource Development, Box 48, Mahé, Republic of Seychelles

Please address correspondence to:

Edwin van Wijngaarden, $\mathrm{PhD}$

Department of Public Health Sciences

University of Rochester School of Medicine and Dentistry

265 Crittenden Blvd, CU 420644

Rochester, NY 14642

Tel.: +1 585275 1985; Fax: +1 5854614532

edwin_van_wijngaarden@urmc.rochester.edu

\section{Acknowledgements:}

This research was supported by grants R01-ES010219, P30-ES01247, R03-ES027514, T32-ES007271, and T32ES007026 from the United States National Institute of Environmental Health Sciences (National Institutes of Health) and in-kind by the Government of the Republic of Seychelles. We acknowledge with thanks the contribution of the nursing and laboratory teams in Seychelles. The study sponsors had no role in the design, collection, analysis, or interpretation of the data; in the writing of the report; or in the decision to submit the article for publication. All authors (Irwin, Yeates, Mulhern, McSorley, Strain, Watson, Grzesik, Thurston, Love, Smith, Mruzek, Shamlaye, Monthy, Myers, Davidson, van Wijngaarden) declare they have no conflicts of interest, financial or otherwise. 


\begin{abstract}
Background

Findings from observational and experimental studies suggest that maternal inflammation during pregnancy is associated with autism spectrum disorder (ASD). We report the first study in humans to examine this association in a large prospective birth cohort.
\end{abstract}

\title{
Methods
}

We studied 788 mother-child pairs from the Seychelles Child Development Study Nutrition Cohort 2. Thirteen inflammatory markers were measured in mothers' serum at 28 weeks' gestation, along with the sum of Thelper 1 (Th1) and 2 (Th2) cytokines. The Social Communication Questionnaire (SCQ) and Social Responsiveness Scale (SRS) were administered at age 7 years to obtain information on ASD phenotype. We evaluated associations between maternal inflammatory markers and ASD phenotype using multivariable linear regression.

\section{Results}

For the SCQ, increased MCP-1 (a chemokine that is upregulated in response to proinflammatory cytokines) was associated with fewer ASD symptoms $(B=-0.40 ; 95 \% \mathrm{CI}=-0.72,-0.09)$. Increased IL-4 (a cytokine that is typically associated with an enhanced anti-inflammatory response) was associated with more ASD symptoms $(B=2.10 ; 95 \% \mathrm{CI}=0.78,3.43)$. For the $\mathrm{SRS}$, higher concentrations of the anti-inflammatory cytokine IL-10 were associated with fewer ASD symptoms $(B=-0.18 ; 95 \% \mathrm{CI}=-0.35,-0.01)$, but only after removal of outliers. No associations were observed for other markers.

\section{Conclusions}

These findings suggest that a shift in the maternal immune balance during pregnancy may be associated with ASD symptomatology. While the use of well-established measures that capture ASD phenotypic variability is a strength of the study, measurement of peripheral immune markers only once during gestation is a limitation. Our results should be confirmed using maternal immune markers measured throughout gestation.

Keywords: Pregnancy; Immune Response; Inflammation; Autism Spectrum Disorder; Cytokines; Chemokines 


\section{Introduction}

Autism spectrum disorder (ASD) is characterized by persistent difficulties of varying severity in social interaction and communication, and restricted or repetitive behaviors and interests [1]. The reported prevalence has increased in the past several decades, with an estimated one in every 59 children having an ASD diagnosis by eight years of age in the United States [2]. The precise etiology of ASD remains unknown [3].

Maternal inflammation during prenatal development is hypothesized to be associated with the pathogenesis and severity of ASD [4]. The immune system works during pregnancy to ensure that the maternal fetal environment is protected from external pathogens while also preventing inflammatory dysregulation that could lead to miscarriage [5]. The balance of pro- versus anti-inflammatory cells shifts throughout pregnancy to support this immune function. In the first trimester, T-helper 1(Th1)-type cytokines are released as part of cell-mediated inflammatory reactions in response to the fetus as a foreign body [6-9]. During the second and early-third trimesters, there is a subsequent downregulation of Th1-type cells in favor of T-helper 2 (Th2)-type immunity in order to prevent rejection of the fetus [6-9]. In the mid- to late-third trimester, there is an upregulation of Th1 cells and downregulation of Th2 cells to promote contractions of the uterus and subsequent delivery of the baby and placenta $[10]$.

Infections or immune dysregulation may harm fetal neural and immune system development if present during critical or sensitive periods [11-13], thereby potentially contributing to the likelihood of ASD [14]. A study of all children born in Denmark from 1980 to 2005 found that hospital admissions due to maternal viral infection in the first trimester and maternal bacterial infection in the second trimester were associated with a significantly increased risk of an ASD diagnosis in their children [15]. Another study using data from a cohort of infants born in California found no overall association between any diagnosis of maternal infection during pregnancy and ASD, but children of women diagnosed with an infection during a hospital admission were more likely to have a diagnosis of ASD [16]. Prenatal exposure to multiple infections was also associated with ASD in that study [16]. A retrospectivecohort study using data from the Western Australian Autism Biological Registry found that maternal immune activation history (i.e., a history of allergies and asthma) during pregnancy was associated with greater severity of childhood ASD symptoms as measured by the Social Responsiveness Scale [17].

Animal models have demonstrated an association between activation of the maternal immune system during pregnancy and possibility of ASD-type behaviors in offspring [18]. Maternal administration of poly(I:C) in 
pregnant mice at gestational day 12.5 resulted in an upregulation of IL-6 that was associated with increased anxiety and social interaction deficits in offspring [19]. Although the role of specific cytokines was not assessed, pregnant mice injected with poly(I:C) in another study had offspring that exhibited high levels of repetitive behaviors and deficits in reciprocal social interaction [20].

Of relevance to the current study, several epidemiologic case-control studies have reported associations between childhood ASD phenotype and specific cytokines and chemokines measured in maternal sera during pregnancy. These studies reported that higher levels of early-pregnancy C-reactive protein (CRP) were associated with increased ASD risk [21] and, conversely, that higher levels of mid-pregnancy CRP were associated with decreased ASD risk [22]. Goines and colleagues [23] found that, among more than one dozen markers assessed, increased concentrations of interferon (IFN)- $\gamma$, interleukin (IL)-4, and IL-5 in maternal sera at 15 to 19 weeks gestation were associated with an increased risk of ASD diagnosis in their children. However, their sample size was limited to 84 children with ASD, 49 children with developmental delay but not ASD, and 159 children without ASD or developmental problems. Authors from the same research group [24] conducted a similar but larger study and reported decreased levels of IFN- $\gamma$, IL-8, and monocyte chemoattractant protein (MCP)-1 in mid-gestational sera of mothers whose children developed ASD without intellectual disability as compared to controls without ASD or developmental problems [24]. Using dried neonatal blood spots [25-29] or mid-gestational amniotic fluid samples $[30,31]$, other case-control studies suggest that the following are associated with an increased probability of ASD: increased MCP-1 [29, 31], increased IL-1 $\beta$ [28], either increased [28, 30] or decreased [25] IL-4, increased IL-10 [30], increased tumor necrosis factor (TNF)- $\alpha$ [30], and decreased IFN- $\gamma$, IL-2, and IL-6 [25].

No studies have assessed the association between maternal inflammatory markers and ASD prospectively, and there are few replicated findings. In addition, studies of more subtle measures capturing variability in ASD phenotype have not been carried out. The current study adds to this limited literature by evaluating data from the Seychelles Child Development Study (SCDS) Nutrition Cohort 2 (NC2). This is a large, well-characterized prospective birth cohort from the Republic of Seychelles with the primary goal of evaluating whether prenatal exposure to methylmercury and nutrients from high fish consumption during pregnancy influences child development [32]. As part of this study we measured 13 prenatal markers of inflammation and obtained data from parent surveys about their child's ASD symptomatology at 7 years of age. We used two widely-accepted ASD screening instruments to characterize the presence and severity of ASD-related symptoms [33, 34], as opposed to 
relying on categorical clinical diagnoses of ASD as in previous studies. Based on existing literature, we hypothesized that increased concentrations of IFN- $\gamma$ [24, 25], MCP-1 [24], and CRP [22] in maternal serum at 28 weeks gestation would be associated with fewer ASD symptoms at 7 years of age. Conversely, we hypothesized that increased concentrations of IL-4, IL-10, and TNF- $\alpha$ would be associated with more ASD symptoms [30].

\section{Methods and Materials}

\section{Study Population}

The SCDS NC2 cohort has been described in detail elsewhere [32]. In short, 1518 pregnant women were recruited from 2008 to 2011 during an early antenatal visit (from 14 weeks' gestation). Inclusion criteria for NC2 included being native Seychellois, being $\geq 16$ years of age, having a singleton pregnancy (if known at recruitment), and having no obvious health concerns [32]. Children were tested for various developmental outcomes at about 20 months and 7 years of age. Developmental testing for this cohort is ongoing, but we examined a subset of participants $(n=788)$ with currently available data on ASD phenotyping measures at 7 years, inflammatory markers in maternal sera at 28 weeks' gestation, and relevant covariates (see "Covariates").

As in previous analyses [32], exclusions were made for factors known to significantly affect neurodevelopment, including birthweight $<1500 \mathrm{~g}(n=4)$, maternal pre- or perinatal complications $(n=1)$, head trauma with loss of consciousness $>1$ minute $(n=2)$, and recurrent afebrile seizures $(n=13)$. We also excluded twins $(n=20)$ because of the potential for differences in the prenatal environment between twins and singletons. After exclusions, the final overall sample size was 748 , with sample sizes ranging from 691 to 702 per analysis due to missing data in inflammatory markers or covariates, and ranging from 687 to 697 after outlier removal (see "Statistical Analyses").

The study was reviewed and approved by the Seychelles Ethics Board and the Research Subjects Review Board at the University of Rochester.

\section{ASD Phenotype Assessment}

The Social Communication Questionnaire (SCQ) [34] and Social Responsiveness Scale (SRS) [33] were administered when cohort children were around 7 years of age $(M=7.19, S D=0.10$, range $=6.25-7.80$ years $)$ to obtain information on ASD phenotype. The SCQ is a 40-item parent rating scale of ASD symptomatology with responses in yes-or-no format. Questions assess lifetime presence of symptoms (items 2 - 19), and symptoms present between the child's fourth and fifth birthdays (items $20-40$ ). The SCQ yields a total score ranging from $0-$ 
39 (the first item is a screener for the use of spoken language and is not included in the total score), with higher scores indicating more ASD symptoms. The average SCQ total score for the current sample was $8.50(S D=4.17$, range: $0-22$ ). The screening cut-off for potential ASD is a total score $\geq 15[34,35]$.

The SRS is a 65-item parent rating scale of ASD symptomatology present over the 6 months prior to administration. Items are rated on a Likert-type scale from 0 ("not true") to 3 ("almost always true"). The SRS yields a total score ranging from $0-195$, with higher scores indicating more ASD-related symptoms. The average SRS total score for the current sample was $48.27(S D=19.17$, range: $6-137)$. A total score $\geq 70$ for males and $\geq 65$ for females is recommended as the cut-off for possible ASD when screening unselected general-population groups [33].

\section{Inflammatory Markers}

Non-fasting blood samples were collected at a mean of 28 weeks of gestation (range: 20.14 - 33.29 weeks, $S D=1.03)$. Samples were collected by antecubital venipuncture into evacuated serum tubes. They were placed on ice and allowed to sit for 30 minutes before being centrifuged at $2500 \mathrm{rpm}$ for 15 minutes. Aliquots were shipped to Ulster at $-80^{\circ} \mathrm{C}$ and stored until analysis. Analysis of serum inflammatory markers took place approximately five years after sample collection and were analyzed at Ulster University using Meso Scale Discovery (MSD) multiplex assay (Meso Scale Diagnostics, LLC). These assays yield greater sensitivity, larger dynamic range, and require less sample volume than traditional methods for immunoassays [36].

The cytokines that were measured for cell-mediated pro-inflammatory reactions included IL-1 $\beta$, IL-2, IFN$\gamma$, and TNF- $\alpha$. The cytokines associated with anti-inflammatory $\beta$-cell activation and antibody production included IL-4, IL-5, and IL-10. Measured cytokines were also used to estimate Th1:Th2, defined as Th1 (sum of IL-1 $\beta$, IL-2, IFN- $\gamma$, and TNF- $\alpha$ ) divided by Th2 (sum of IL-4, IL-5, IL-10, and IL-6). This ratio provides an approximation of the balance of cytotoxic (Th1) versus humoral immune (Th2) responses. IL-6, which has both pro- and antiinflammatory roles [37], was also measured. Additional markers included CRP; the chemokines MCP-1 and thymus and activation regulated chemokine (TARC); and the angiogenesis markers soluble fms-like tyrosine kinase (sFlt)-1 and vascular endothelial growth factor (VEGF)-D. Individual biomarkers were analyzed as continuous variables measured in $\mathrm{pg} / \mathrm{mL}$, except CRP which was measured in $\mathrm{mg} / \mathrm{L}$.

Values below the limit of detection for each marker were imputed as $\operatorname{LLOD} / \sqrt{2}$, as recommended by others [38]. Two markers had a substantial amount (>20\%) of undetectable values: IL-2 and IL-4. Due to their relatively low concentrations, excluding IL-2 and IL-4 from the Th1, Th2 and Th1:Th2 variables had little impact on 
associations studied with developmental outcomes at 20 months in this cohort [Irwin et al. submitted], and thus these markers were included both as individual variables and within the Th1, Th2, and Th1:Th2 variables in the current analyses.

\section{Covariates}

Our regression models adjusted for demographic covariates based on their known or suspected relationship with ASD phenotype as identified in a recent meta-analysis of prenatal, perinatal, and postnatal factors associated with ASD [39]: maternal age, child sex, Hollingshead socioeconomic status (SES), and parity (1 child versus 2+ children). Information on paternal age was not available. Both maternal age and Hollingshead SES (possible range 8-66, with higher scores indicating higher SES) were included as continuous variables.

\section{Statistical Analyses}

First, we calculated measures of central tendency and variance to describe demographics, ASD phenotype, and inflammatory markers in mothers and children. To correct for significant skewness in the inflammatory marker variables, each was $\log _{\mathrm{e}}$-transformed after adding a constant of one to prevent taking the natural logarithm of zero. In addition, the SRS total score was square-root transformed to correct for significant skewness. Transformed variables were used in all analyses, with the exception of the descriptive analyses presented in Table 1.

Primary analyses consisted of 16 separate multivariable regression models, separately for SCQ and SRS. We examined each of the 13 individual biomarkers (IL-1 $\beta$, IL-2, IFN- $\gamma$, TNF- $\alpha$, IL-4, IL-5, IL-6, IL-10, MCP-1, TARC, sFlt-1, VEGF-D, CRP) as well as total Th1, Th2, and Th1:Th2 as continuous predictors. In secondary analyses we evaluated the independent association for each individual marker after accounting for other markers. We also performed regression with all 13 individual markers in one model, and total Th1 and Th2 together in another model. Results of the secondary analyses were identical in interpretation to the results of the primary analyses, thus only results of the primary analyses are presented below.

Prior to running the analysis, each model was examined for the presence of influential points (Cook's distances larger than 0.50 ) or statistical outliers (standardized residuals in excess of \pm 3 ), as has been done previously [40]. There were no influential points, but 4-6 statistical outliers were identified depending on the model.

Observations that were statistical outliers had significantly higher mean SRS $(M=98.17, S D=30.31)$ and SCQ $(M=$ $17.00, S D=3.88)$ scores compared to the rest of the sample. Each regression model was then also run without its respective outliers included. 
For each analysis, a $p$ value less than 0.05 was considered statistically significant. All statistical analyses were conducted using SAS (v9.4, SAS Institute Inc., Cary, NC, USA).

\section{Results}

Summary statistics for the participants and variables included in the study are presented in Tables 1 and 2. Figures 1 and 2 include the results of the analyses investigating whether each individual inflammatory marker variable, as well as Th1, Th2, and Th1:Th2 is associated with the SCQ total score (Figure 1) and the SRS total score (Figure 2), each in separate models.

For the SCQ, higher concentrations of MCP-1, a chemokine upregulated in response to pro-inflammatory cytokines, were associated with fewer ASD symptoms, as hypothesized. Also as hypothesized, higher concentrations of IL-4, a cytokine typically associated with an enhanced anti-inflammatory response, were associated with more ASD symptoms. No other inflammatory marker variables (IL-1 $\beta$, IL-2, IFN- $\gamma$, TNF- $\alpha$, IL-5, IL-10, IL-6, TARC, sFlt-1, VEGF-D, CRP, Th1, Th2, Th:Th2) were significantly associated with SRS or SCQ total scores. After removal of outliers, model results were similar to those using the full sample, although the association between higher concentrations of IL-10 and fewer ASD symptoms measured by the SRS became statistically significant $(B=$ $-0.18 ; 95 \% \mathrm{CI}=-0.35,-0.01)$.

Across all models, sex and Hollingshead SES were associated with SRS and SCQ total scores; being female and mothers having higher SES were associated with fewer ASD symptoms. Mothers' age at enrollment and parity were not associated with SRS or SCQ scores in any models.

\section{Discussion}

The current study evaluated whether circulating maternal inflammatory markers at 28 weeks' gestation are associated with child ASD phenotype at 7 years. We hypothesized that increased concentrations of CRP, IFN- $\gamma$, and MCP-1 would be associated with reduced ASD symptomatology and increased IL-4, IL-10, and TNF- $\alpha$ concentrations would be associated with elevated ASD symptomatology. These hypotheses were confirmed for MCP-1 and IL-4, but not for CRP, IFN- $\gamma$, IL-10, and TNF- $\alpha$. Contrary to our hypothesis, higher concentrations of IL-10 were associated with fewer ASD symptoms, although this association was only statistically significant after removal of outliers.

MCP-1 is a chemokine that plays a key role in the polarization of cells toward a Th2 phenotype through its recruitment of activated T cells, monocytes, and basophils to sites of inflammation or tissue injury [41]. A study 
using mice found that activation of the maternal innate immune system via poly(I:C) triggers an upregulation of MCP-1 that is also evident in the fetal brain, which could theoretically impair the brain's ability to form neuronal connections [42]. Nevertheless, other research has found that MCP-1 supports a neuroprotective role by causing proliferation of microglia that protect the central nervous system from pathogens [43]. MCP-1 was associated with reduced ASD symptomatology, consistent with prior research on children with ASD without intellectual disability [24]. This finding suggests that the association may also extend to children unselected for ASD diagnostic status who exhibit the full range of phenotypic variability across the autism spectrum.

A Th2-dominant profile in normal pregnancy is thought to reduce the maternal response to fetal antigens, in particular when IL-4 and IL-10 concentrations are especially elevated [9]. The primary function of IL-4 in pregnancy is the activation of B-cells and suppression of Th1 cells, and the primary function of IL-10 in pregnancy is to inhibit macrophage cytokine production [44]. IL-4 is associated with an enhanced Th2 response which in a normal situation is associated with antibody production and the clearance of antigens. However, if it is maintained or occurs where the antigen is a self-antigen, it could serve to prolong inflammation [45]. In our sample, increased IL-4 concentrations were associated with elevated ASD symptomatology, whereas increased IL-10 concentrations were associated with reduced ASD symptomatology. This is possibly reflective of an association between immune dysregulation and ASD symptomatology. Our initial hypotheses that increased IL-4 and IL-10 would be associated with elevated ASD symptomatology were based on findings that suggested a higher probability of ASD diagnosis with increased IL-10 and IL-4 in amniotic fluid [30]. However, a study of normative cytokine concentrations in pregnancy found that mid-gestational concentrations of IL-10 were detectable in only $34 \%$ of amniotic fluid samples, whereas they were detectable in $100 \%$ of maternal serum samples [44]. Consequently, findings using IL10 concentrations in amniotic fluid may not be comparable to those using maternal sera. Concentrations of IL-4, however, were detectable in $100 \%$ of both maternal serum and amniotic fluid samples in that study [44].

This study has important strengths, in particular its use of a large prospective birth cohort of children, the relatively large panel of inflammatory markers, and the use of continuous measures of ASD symptomatology as opposed to clinical ASD diagnostic status. We used two widely-accepted ASD screening instruments to characterize the presence and severity of ASD-related symptoms [33, 34], examining ASD features along a continuum in order to capture the full range of phenotypic variability $[46,47]$. The use of these continuous measures in a prospective birth cohort also allowed us to have roughly equivalent numbers of males and females in the sample. Females are less 
likely than males to receive an ASD diagnosis even when exhibiting equivalent high levels of autistic-like traits [48], and are therefore underrepresented in observational studies of ASD or related phenotypes. Finally, we were able to confirm known associations for covariates included in our analyses, further increasing the confidence in our findings. For example, the significant association with socioeconomic status is in line with recent research suggesting that both SRS and SCQ scores are impacted by maternal education and family income [49]. Also, sex differences in ASD symptomatology were as expected based on findings from general population groups indicating that males tend to have higher scores on both the SRS[33] and SCQ[50] compared to females.

Our study also has several limitations. Concentrations of inflammatory markers are known to fluctuate throughout pregnancy $[8,9]$; therefore concentrations at 28 weeks gestation likely vary from concentrations at other time points that were not measured in the current study. Furthermore, although we excluded mothers and children with certain clinical conditions from the analysis, we lacked information on whether the mothers had particular inflammatory or autoimmune conditions, which might impact concentrations of inflammatory markers. In addition, measurable levels of inflammatory markers can be affected by the time samples spent in storage (in this study, approximately five years), for example due to cytokine degradation [51]. However, all samples in the current study underwent the same conditions of freezing, storage, and thawing, and we would not expect the impact of storage on inflammatory marker concentrations to vary based on ASD phenotype or other variables in the model.

In conclusion, our findings in a large prospective cohort suggest that a shift in the maternal immune balance during pregnancy may be associated with ASD symptomatology. Our results should be confirmed using longitudinal measurements of maternal inflammatory markers throughout gestation. 


\section{References}

1. American Psychiatric Association (2013) Diagnostic and statistical manual of mental disorders (5th ed). American Psychiatric Association, Arlington, VA

2. Baio J, Wiggins LD, Christensen DL, et al (2018) Prevalence of Autism Spectrum Disorder Among Children Aged 8 Years - Autism and Developmental Disabilities Monitoring Network, 11 Sites, United States, 2014. Morb Mortal Wkly Rep Surveill Summ 67:1-23 . doi: http://dx.doi.org/10.15585/mmwr.ss6706a1

3. Lyall K, Croen L, Daniels J, et al (2017) The Changing Epidemiology of Autism Spectrum Disorders. Annu Rev Public Health 38:81-102 . doi: 10.1146/annurev-publhealth-031816-044318

4. Gladysz D, Krzywdzińska A, Hozyasz KK (2018) Immune Abnormalities in Autism Spectrum DisorderCould They Hold Promise for Causative Treatment? Mol Neurobiol 1-49 . doi: 10.1007/s12035-017-0822-x

5. Aghaeepour N, Ganio EA, Mcilwain D, et al (2017) An immune clock of human pregnancy. Sci Immunol 2:eaan2946 . doi: 10.1126/sciimmunol.aan2946

6. Mosmann TR, Coffman RL (1989) ThI and Th2 CELLS: Different Patterns of Lymphokine Secretion Lead to Different Functional Properties. Ann Rev Immuno 7:145-73

7. Saito S, Sakai M, Sasaki Y, et al (1999) Quantitative analysis of peripheral blood Th0, Th1, Th2 and the Th1:Th2 cell ratio during normal human pregnancy and preeclampsia. Clin Exp Immunol 117:550-555 . doi: 10.1046/j.1365-2249.1999.00997.x

8. Sargent IL, Borzychowski AM, Redman CWG (2006) NK cells and human pregnancy - an inflammatory view. Trends Immunol 27:399-404 . doi: 10.1016/j.it.2006.06.009

9. Wegmann TG, Lin H, Guilbert L, Mosmann TR (1993) Bidirectional cytokine interactions in the maternalfetal relationship: Is successful pregnancy a TH2 phenomenon? Immunol Today 14:353-356 . doi: 10.1016/0167-5699(93)90235-D

10. Mor G, Cardenas I, Abrahams V, Guller S (2011) Inflammation and pregnancy: The role of the immune system at the implantation site. Ann N Y Acad Sci 1221:80-87 . doi: 10.1111/j.1749-6632.2010.05938.x

11. Estes ML, McAllister AK (2016) Maternal immune activation: Implications for neuropsychiatric disorders. Science (80- ) 353:772 LP-777 . doi: 10.1126/science.aag3194

12. Knuesel I, Chicha L, Britschgi M, et al (2014) Maternal immune activation and abnormal brain development 
across CNS disorders. Nat Rev Neurol 10:643-660 . doi: 10.1038/nrneurol.2014.187

13. Racicot K, Kwon JY, Aldo P, et al (2014) Understanding the complexity of the immune system during pregnancy. Am J Reprod Immunol 72:107-116 . doi: 10.1111/aji.12289

14. Bilbo SD, Block CL, Bolton JL, et al (2018) Beyond infection - Maternal immune activation by environmental factors, microglial development, and relevance for autism spectrum disorders. Exp. Neurol. 299:241-251

15. Atladóttir HÓ, Thorsen P, Østergaard L, et al (2010) Maternal infection requiring hospitalization during pregnancy and autism spectrum disorders. J Autism Dev Disord 40:1423-1430 . doi: 10.1007/s10803-0101006-y

16. Zerbo O, Qian Y, Yoshida C, et al (2015) Maternal Infection During Pregnancy and Autism Spectrum Disorders. J Autism Dev Disord 45:4015-4025 . doi: 10.1007/s10803-013-2016-3

17. Patel S, Masi A, Dale RC, et al (2017) Social impairments in autism spectrum disorder are related to maternal immune history profile. Mol Psychiatry. doi: 10.1038/mp.2017.201

18. Pardo CA, Meffert MK (2018) Animal models in autism research: The legacy of Paul H. Patterson. Exp. Neurol. 299:197-198

19. Smith SEP, Li J, Garbett K, et al (2007) Maternal immune activation alters fetal brain development through interleukin-6. J Neurosci 27:10695-10702 . doi: 10.1523/JNEUROSCI.2178-07.2007

20. Malkova N V., Yu CZ, Hsiao EY, et al (2012) Maternal immune activation yields offspring displaying mouse versions of the three core symptoms of autism. Brain Behav Immun. doi: 10.1016/j.bbi.2012.01.011

21. Brown AS, Sourander A, Hinkka-Yli-Salomäki S, et al (2014) Elevated maternal C-reactive protein and autism in a national birth cohort. Mol Psychiatry 19:259-264 . doi: 10.1038/mp.2012.197

22. Zerbo O, Traglia M, Yoshida C, et al (2016) Maternal mid-pregnancy C-reactive protein and risk of autism spectrum disorders: The early markers for autism study. Transl Psychiatry 6:e783 . doi: 10.1038/tp.2016.46

23. Goines PE, Croen LA, Braunschweig D, et al (2011) Increased midgestational IFN- $\gamma$, IL-4 and IL-5 in women bearing a child with autism: A case-control study. Mol Autism 2: . doi: 10.1186/2040-2392-2-13

24. Jones KL, Croen LA, Yoshida CK, et al (2017) Autism with intellectual disability is associated with increased levels of maternal cytokines and chemokines during gestation. Mol Psychiatry 22:273-279 . doi: $10.1038 / \mathrm{mp} .2016 .77$ 
25. Abdallah MW, Larsen N, Mortensen EL, et al (2012) Neonatal levels of cytokines and risk of autism spectrum disorders: An exploratory register-based historic birth cohort study utilizing the Danish Newborn Screening Biobank. J Neuroimmunol 252:75-82 . doi: 10.1016/j.jneuroim.2012.07.013

26. Abdallah MW, Larsen N, Grove J, et al (2013) Neonatal chemokine levels and risk of autism spectrum disorders: Findings from a Danish historic birth cohort follow-up study. Cytokine 61:370-376 . doi: 10.1016/j.cyto.2012.11.015

27. Abdallah MW, Mortensen EL, Greaves-Lord K, et al (2013) Neonatal levels of neurotrophic factors and risk of autism spectrum disorders. Acta Psychiatr Scand 128:61-69 . doi: 10.1111/acps.12020

28. Krakowiak P, Goines PE, Tancredi DJ, et al (2017) Neonatal Cytokine Profiles Associated With Autism Spectrum Disorder. Biol Psychiatry 81:442-451 . doi: 10.1016/j.biopsych.2015.08.007

29. Zerbo O, Yoshida C, Grether JK, et al (2014) Neonatal cytokines and chemokines and risk of autism spectrum disorder: The Early Markers for Autism (EMA) study: A case-control study. J Neuroinflammation 11:1-9 . doi: 10.1186/1742-2094-11-113

30. Abdallah MW, Larsen N, Grove J, et al (2013) Amniotic fluid inflammatory cytokines: Potential markers of immunologic dysfunction in autism spectrum disorders. World J Biol Psychiatry 14:528-538 . doi: $10.3109 / 15622975.2011 .639803$

31. Abdallah MW, Larsen N, Grove J, et al (2012) Amniotic fluid chemokines and autism spectrum disorders: An exploratory study utilizing a Danish Historic Birth Cohort. Brain Behav Immun 26:170-176 . doi: 10.1016/j.bbi.2011.09.003

32. Strain JJ, Yeates AJ, van Wijngaarden E, et al (2015) Prenatal exposure to methyl mercury from fish consumption and polyunsaturated fatty acids: Associations with child development at 20 mo of age in an observational study in the Republic of Seychelles. Am J Clin Nutr 101:530-537 . doi: 10.3945/ajen.114.100503

33. Constantino JN, Gruber CP (2005) Social Responsiveness Scale (SRS): Manual. Western Psychological Services, Los Angeles, CA

34. Rutter M, Bailey A, Lord C (2003) Social Communication Questionnaire. Manual. Western Psychological Services, Los Angeles, CA

35. Berument SK, Rutter M, Lord C, et al (1999) Autism screening questionnaire: Diagnostic validity. Br J 
Psychiatry 175:444-451 . doi: 10.1192/bjp.175.5.444

36. Fu Q, Zhu J, Van Eyk JE (2010) Comparison of multiplex immunoassay platforms. Clin Chem 56:314-318 . doi: 10.1373/clinchem.2009.135087

37. Diehl S, Rincón M (2002) The two faces of IL-6 on Th1/Th2 differentiation. Mol Immunol 39:531-536 . doi: 10.1016/S0161-5890(02)00210-9

38. Ogden TL (2010) Handling results below the level of detection. Ann. Occup. Hyg. 54:255-256

39. Wang C, Geng H, Liu W, Zhang G (2017) Prenatal, perinatal, and postnatal factors associated with autism: A meta-analysis. Med (United States) 96:e6696 . doi: 10.1097/MD.0000000000006696

40. van Wijngaarden E, Thurston SW, Myers GJ, et al (2013) Prenatal methyl mercury exposure in relation to neurodevelopment and behavior at 19 years of age in the Seychelles Child Development Study. Neurotoxicol Teratol 39:19-25 . doi: 10.1016/j.ntt.2013.06.003

41. Deshmane SL, Kremlev S, Amini S, Sawaya BE (2009) Monocyte Chemoattractant Protein-1 (MCP-1): An Overview. J Interf Cytokine Res 29:313-326 . doi: 10.1089/jir.2008.0027

42. Arrode-Brusés G, Brusés JL (2012) Maternal immune activation by poly I:C induces expression of cytokines IL-1 $\beta$ and IL-13, chemokine MCP-1 and colony stimulating factor VEGF in fetal mouse brain. J Neuroinflammation 9:83 . doi: 10.1186/1742-2094-9-83

43. Hinojosa AE, Garcia-Bueno B, Leza JC, Madrigal JLM (2011) CCL2/MCP-1 modulation of microglial activation and proliferation. J Neuroinflammation 8: . doi: 10.1186/1742-2094-8-77

44. Chow SSW, Craig ME, Jones CA, et al (2008) Differences in amniotic fluid and maternal serum cytokine levels in early midtrimester women without evidence of infection. Cytokine 44:78-84 . doi: 10.1016/j.cyto.2008.06.009

45. Murphy K, Weaver C (2016) Janeway’s Immunobiology, 9th ed. Garland Science, New York, NY

46. Lundström S, Chang Z, Råstam M, et al (2012) Autism spectrum disorders and autisticlike traits: Similar etiology in the extreme end and the normal variation. Arch Gen Psychiatry 69:46-52 . doi:

10.1001/archgenpsychiatry.2011.144

47. Spiker D, Lotspeich LJ, Dimiceli S, et al (2002) Behavioral phenotypic variation in autism multiplex families: Evidence for a continuous severity gradient. Am J Med Genet - Neuropsychiatr Genet 114:129136 . doi: 10.1002/ajmg.10188 
48. Dworzynski K, Ronald A, Bolton P, Happé F (2012) How different are girls and boys above and below the diagnostic threshold for autism spectrum disorders? J Am Acad Child Adolesc Psychiatry 51:788-797 . doi: 10.1016/j.jaac.2012.05.018

49. Moody EJ, Reyes N, Ledbetter C, et al (2017) Screening for Autism with the SRS and SCQ: Variations across Demographic, Developmental and Behavioral Factors in Preschool Children. J Autism Dev Disord 47:3550-3561 . doi: 10.1007/s10803-017-3255-5

50. Evans SC, Boan AD, Bradley C, Carpenter LA (2018) Sex/Gender Differences in Screening for Autism Spectrum Disorder: Implications for Evidence-Based Assessment. J Clin Child Adolesc Psychol 1-15 . doi: $10.1080 / 15374416.2018 .1437734$

51. de Jager W, Bourcier K, Rijkers GT, et al (2009) Prerequisites for cytokine measurements in clinical trials with multiplex immunoassays. BMC Immunol 10:52 . doi: 10.1186/1471-2172-10-52 


\section{Table and Figure Titles/Legends:}

Table 1: Summary statistics for ASD phenotype at 7 years of age, and model covariates.

Note. ASD: Autism Spectrum Disorder; SRS: Social Responsiveness Scale; SCQ: Social Communication Questionnaire; SES: socioeconomic status.

Table 2: Summary statistics for maternal inflammatory markers at 28 weeks gestation.

Note. LOD: limit of detection; IL-1 $\beta$ : Interleukin-1 $\beta$; IL-2: Interleukin-2; IFN- $\gamma$ : Interferon- $\gamma$; TNF- $\alpha$ : Tumor Necrosis Factor- $\alpha$; Th1: Sum of IL-1 $\beta$, IL-2, IFN- $\gamma$, TNF- $\alpha$; IL-4: Interleukin-4; IL-5: Interleukin-5; IL-10: Interleukin-10; Th2: Sum of IL-4, IL-5, IL-10; IL-6: Interleukin-6; MCP-1: Monocyte Chemoattractant Protein-1; TARC: Thymus and Activation Regulated Chemokine; sFlt-1: Soluble Fms-like Tyrosine Kinase1; VEGF-D: Vascular Endothelial Growth Factor-D; CRP: C-reactive protein; ASD: Autism Spectrum Disorder; SRS: Social Responsiveness Scale; SCQ: Social Communication Questionnaire. Serum biomarkers measured in $\mathrm{pg} / \mathrm{mL}$, except CRP which was measured in $\mathrm{mg} / \mathrm{L}$. Descriptive analyses presented are using raw variables prior to transformation. 
Figure 1: Primary main effects models for continuous maternal gestational inflammatory markers at 28 weeks and the Social Communication Questionnaire at 7 years of age.

Note. Primary analyses include individual markers, Th1, and Th2 in separate models. Estimated regression coefficients and corresponding $95 \%$ confidence intervals are shown, after adjustment for the following covariates: maternal age at enrollment, child sex, parity, and Hollingshead socioeconomic status. ${ }^{*} p<0.05 ;{ }^{* *} p<0.01$. Th1: Sum of IL-1 $\beta$, IL-2, IFN- $\gamma$, TNF- $\alpha$; Th2: Sum of IL-4, IL-5, IL-10. All inflammatory marker variables were log-transformed, as outlined in text.

Figure 2: Primary main effects models for continuous maternal gestational inflammatory markers at 28 weeks and the Social Responsiveness Scale at 7 years of age.

Note. Primary analyses include individual markers, Th1, and Th2 in separate models. Estimated regression coefficients and corresponding $95 \%$ confidence intervals are shown, after adjustment for the following covariates: maternal age at enrollment, child sex, parity, and Hollingshead socioeconomic status. Th1: Sum of IL-1 $\beta$, IL-2, IFN- $\gamma$, TNF- $\alpha$; Th2: Sum of IL-4, IL-5, IL-10. All inflammatory marker variables were log-transformed, as outlined in text. 


\begin{tabular}{lccccc}
\hline & $n$ & $M(S D)$ or $n(\%)$ & Minimum & Median & Maximum \\
\hline ASD Phenotype Measures & & & & & \\
SRS total score & 747 & $48.27(19.17)$ & 6 & 47.00 & 137 \\
SCQ total score & 746 & $8.50(4.17)$ & 0 & 8 & 22 \\
Covariates & & & & \\
Child sex (male or female) & 748 & $400(53.5 \%)$ males & & & \\
Mother’s age at enrollment & 747 & $26.61(6.14)$ & 16.03 & 25.72 & 46.56 \\
Parity (first child vs. 2+ children) & 739 & $372(50.3 \%)$ first child & & & \\
Hollingshead SES & 742 & $33.10(10.60)$ & 12.50 & 32.00 & 63.00 \\
\hline
\end{tabular}




\begin{tabular}{|c|c|c|c|c|c|}
\hline & $n$ & $M(S D)$ or $n(\%)$ & Minimum & Median & Maximum \\
\hline \multicolumn{6}{|c|}{ Maternal Serum Biomarkers } \\
\hline \multicolumn{6}{|l|}{ Th1 } \\
\hline IL-1 $\beta$ & 718 & $0.26(0.58)$ & $1.00 \mathrm{E}-04$ & 0.09 & 11.91 \\
\hline IL-2 & 718 & $0.24(0.60)$ & $76.3 \% \leq \mathrm{LOD}(0.09)$ & 0.62 & 9.97 \\
\hline IFN- $\gamma$ & 718 & $5.43(20.30)$ & 0.04 & 2.48 & 449.91 \\
\hline TNF- $\alpha$ & 718 & $5.98(8.54)$ & 0.03 & 4.46 & 125.57 \\
\hline$\sum$ Th1 & 718 & $11.90(22.91)$ & 0.21 & 7.75 & 456.99 \\
\hline \multicolumn{6}{|l|}{ Th2 } \\
\hline IL-4 & 718 & $0.14(0.64)$ & $77.0 \% \leq \mathrm{LOD}(0.02)$ & 0.24 & 11.83 \\
\hline IL-5 & 718 & $1.40(2.61)$ & $1.59 \mathrm{E}-03$ & 0.72 & 31.96 \\
\hline IL-10 & 718 & $1.21(1.88)$ & $1.02 \mathrm{E}-03$ & 0.73 & 25.72 \\
\hline$\sum \mathrm{Th} 2$ & 718 & $2.74(3.51)$ & 0.05 & 1.93 & 32.36 \\
\hline \multicolumn{6}{|l|}{ Other } \\
\hline Th1:Th2 & 718 & $8.32(14.05)$ & 0.04 & 4.79 & 220.00 \\
\hline IL-6 & 718 & $0.83(1.40)$ & $3.43 \mathrm{E}-03$ & 0.42 & 14.65 \\
\hline MCP-1 & 718 & $66.61(53.22)$ & 0.02 & 58.41 & 903.61 \\
\hline TARC & 718 & $112.20(186.65)$ & 0.12 & 80.85 & 2541.00 \\
\hline sFlt-1 & 718 & $2012.68(1093.85)$ & 324.63 & 1772.03 & 8523.58 \\
\hline VEGF-D & 718 & $805.46(403.84)$ & 44.71 & 725.71 & 2814.71 \\
\hline CRP & 708 & $3.31(2.64)$ & 0 & 2.50 & 9.73 \\
\hline
\end{tabular}




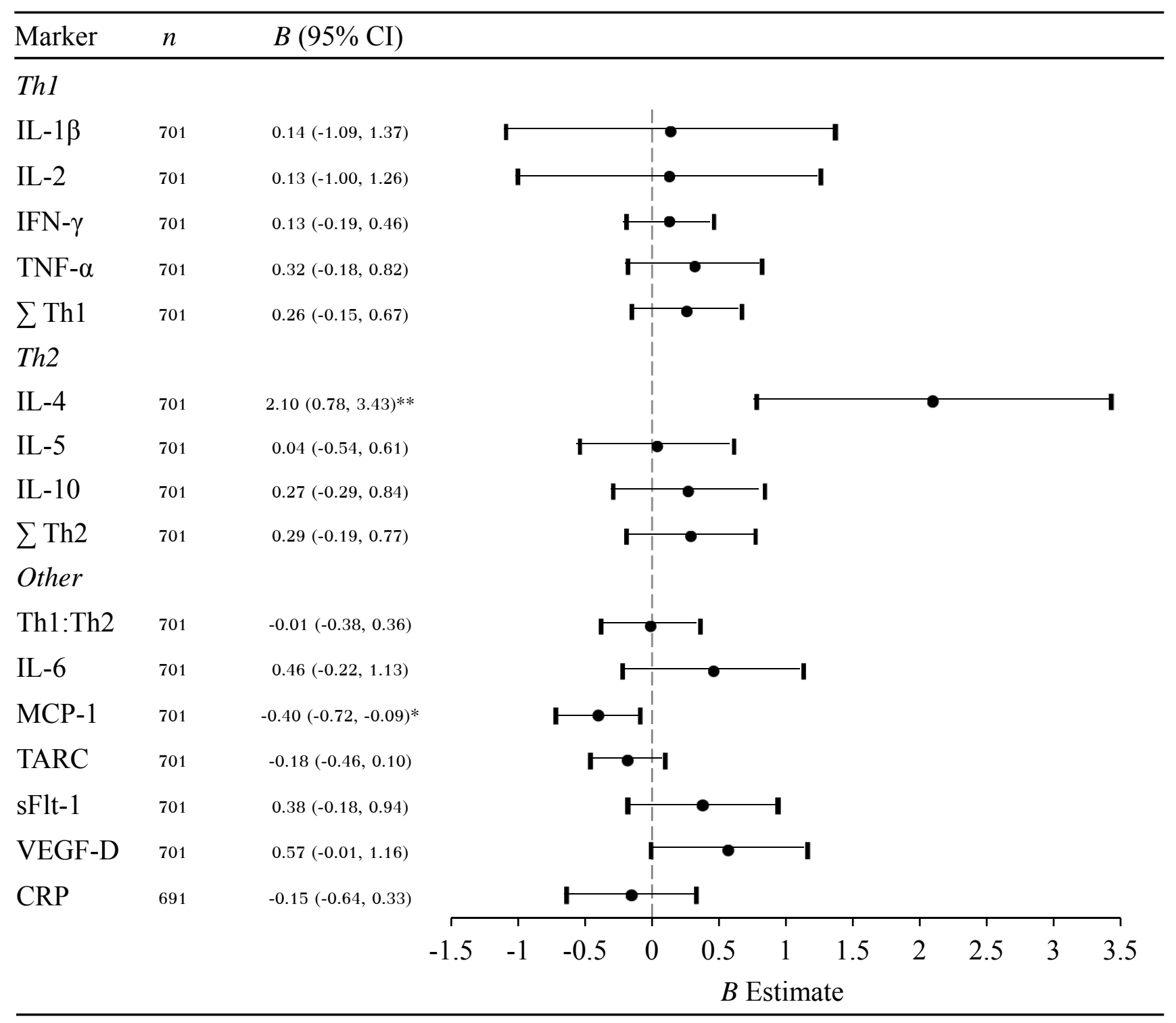




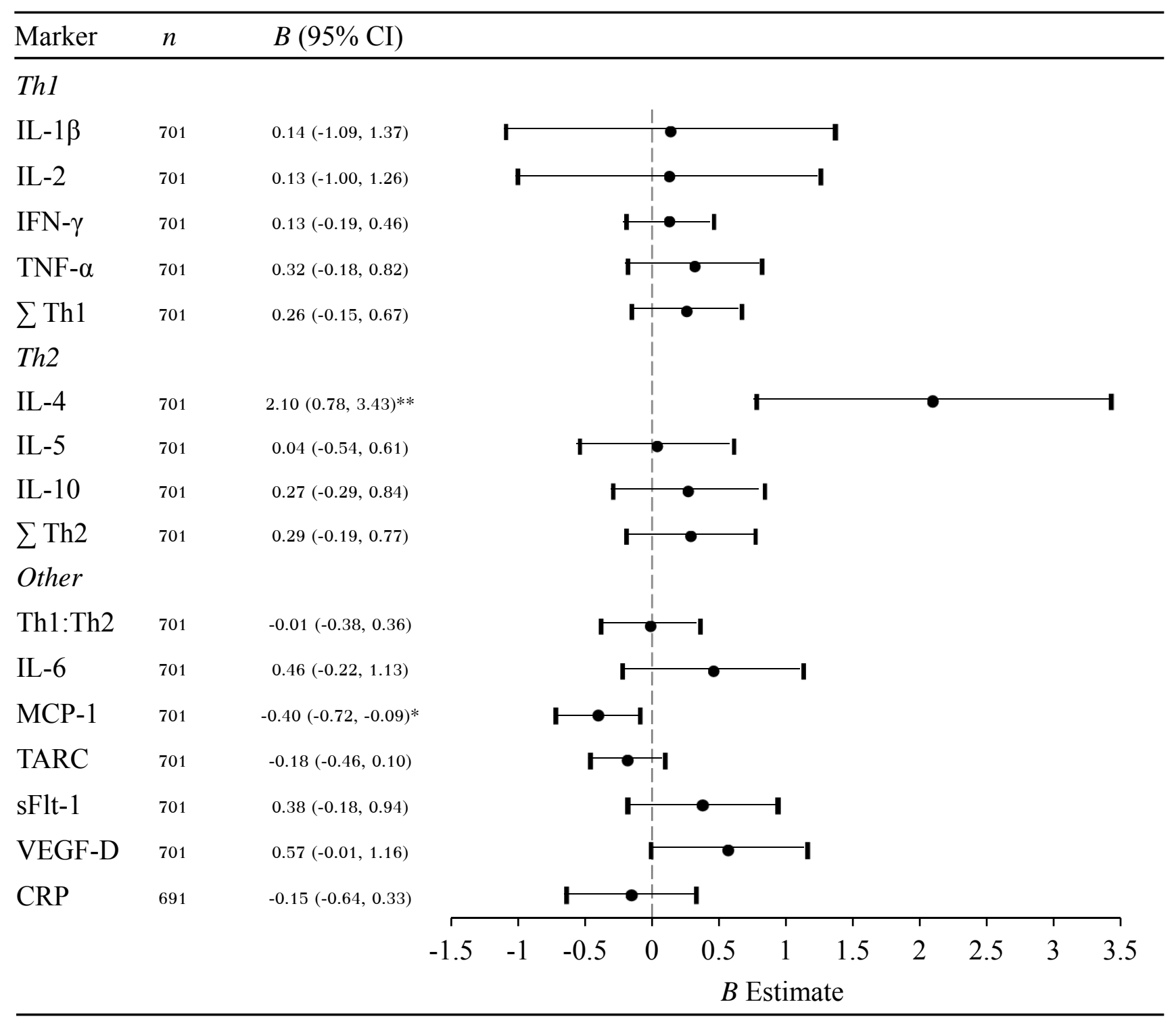

